Abanico Veterinario. Enero-Diciembre 2021; 11:1-11. http://dx.doi.org/10.21929/abavet2021.22

Caso Clínico. Recibido: 20/11/2020. Aceptado: 21/04/2021. Publicado: 10/05/2021. Clave:2020-92.

\title{
Metaplasia cartilaginosa en aurícula derecha de ovino, atribuible al consumo de Trisetum flavescens
}

Cartilaginous metaplasia in the right atrium of sheep, attributable to the consumption

of Trisetum flavescens

\section{Valente Velázquez-Ordoñez ${ }^{1 \text { ID }}$, Adrián Zaragoza-Bastida ${ }^{2^{*}}{ }^{1 D}$, Nallely Rivero- Pérez $^{2}$ ID, Lucia Delgadillo-Ruiz ${ }^{3}$ ID, Perla Gallegos-Flores ${ }^{3}$ ID, Benjamín Valladares-Carranza ${ }^{\star * 1}$ ID}

${ }^{1}$ Facultad de Medicina Veterinaria y Zootecnia. Universidad Autónoma del Estado de México. ${ }^{2}$ Área Académica de Medicina Veterinaria y Zootecnia. Universidad Autónoma del Estado de Hidalgo. ${ }^{3}$ Unidad Académica de Ciencias Biológicas. Universidad Autónoma de Zacatecas. *Autor responsable: Adrián Zaragoza-Bastida. ${ }^{* *}$ Autor de correspondencia: Benjamín Valladares-Carranza. El Cerrillo Piedras Blancas, Estado de México. CP. 50295. Toluca, Estado de México, México. 7222965549 o 722 2966382.vvvo@uaeh.edu.mx, adrian_zaragoza@uaeh.edu.mx, nallely_rivero@uaeh.edu.mx, delgadillolucia@gmail.com, perla_gf17@hotmail.com, bvalladaresc@uaemex.mx

\section{Resumen}

Se presenta el caso de un ovino de 2.5 años que fue enviado para valoración diagnóstica a la necropsia, donde se encontró degeneración mucoide de grasa coronaria, adherencias pleurales, congestión hipostática, hemoperitoneo, distención de retículo, rumen, omaso y abomaso. El hallazgo más significativo fue la apariencia blanquecina y dura al tacto de la aurícula derecha. La muestra de aurícula se sometió a descalcificación para realizar el corte e histopatología. En la histología del corte realizado se observó: zonas extensas de metaplasia cartilaginosa, tumefacción y ondulación de fibras musculares cardiacas, infiltración grasa entre fibras cardiacas, así como figuras compatibles con Sarcocystis. La bioquímica clínica indicó hiperfosfatemia e hipermagnesemia. El proceso encontrado en el ovino evaluado fue metaplasia cartilaginosa de aurícula derecha, asociado a la ingesta de Trisetum flavescens. En las diferentes especies animales la probabilidad de intoxicación progresiva por el consumo de plantas tóxicas es un riesgo que puede darse en cualquier sistema de producción animal.

Palabras clave: metaplasia cartilaginosa, aurícula, ovino.

\begin{abstract}
The case of a 2.5-year-old sheep sent for diagnostic evaluation is presented. At the necropsy, mucoid degeneration of coronary fat, pleural adhesions, hypostatic congestion, hemoperitoneum, reticulum, rumen, omasum, and abomasum distention were found. The most significant finding was the whitish, hard-to-touch appearance of the right atrium. The atrial sample was decalcified for sectioning and histopathology study. The histology of the section performed revealed: extensive areas of cartilaginous metaplasia, swelling, and undulation of cardiac muscle fibers, fatty infiltration between cardiac fibers, as well as figures compatible with Sarcocystis. The clinical chemistry indicated hyperphosphatemia and hypermagnesemia. According to the process for the sheep diagnostic evaluation, cartilaginous metaplasia of the right atrium, associated with the ingestion of Trisetum flavescens was found. In the different animal species, the probability of progressive poisoning from the consumption of toxic plants is a risk that can occur in any animal production system.
\end{abstract}

Keywords: cartilaginous metaplasia, atrium, sheep. 


\section{INTRODUCCIÓN}

Las plantas calcigénicas se encuentran entre los vegetales más nocivos para los animales en el mundo (Odriozola et al., 2018; Zanuzzi et al., 2008), la naturaleza química de los agentes tóxicos contenidos inducen la calcinosis, el principal principio activo es un glucósido esteroideo que se hidroliza en el intestino, el rumen y otros tejidos y libera el fragmento esteroideo, el cual es en la mayoría de los casos el 1,25 $(\mathrm{OH})_{2} \mathrm{D}_{3}$ (Waser et al., 1983; Dallorso et al., 2001; Wu y Sun, 2011). El exceso de vitamina $\mathrm{D}$ estimula la síntesis de proteína de unión a calcio (CaBP) y la absorción de calcio y fosfato, producen hipercalcemia e hiperfosfatemia (Sun, 2010; Dirksen et al., 2003). El mineral absorbido en exceso no puede ser metabolizado, se deposita en los tejidos blandos y produce calcinosis (Mello, 2003; Zanuzzi et al., 2012).

La calcinosis causada por una intoxicación vegetal crónica, es una enfermedad bien conocida en Argentina, Brasil, Paraguay y Uruguay, ha recibido distintos nombres, como "enteque ossificans", "bichoquera", espichamiento" y "enteque seco" (Odriozola et al., 2018; Machado et al., 2020). Cuadros similares se han descrito en diversas regiones del mundo afectando a animales en pastoreo; en todos ellos, la deposición de sales de calcio en los tejidos blandos, se acompaña de un severo deterioro físico y decaimiento de los animales. Hasta el momento se han descrito seis plantas que inducen calcinosis sistémica por intoxicación crónica: Cestrum diurnum, Nierembergia veitchii, Solanum glaucophyllum, Solanum torvum, Stenotaphrum secundatum y Trisetum flavescens (Grabner et al., 1985; Braun et al., 2000).

Trisetum flavescens causa una calcinosis enzoótica en Alemania y en Austria, además de presentarse en Sudamérica. La ingestión de Cestrum diurnum afecta al ganado bovino y equino en Florida EUA. Solanum trovum ha sido asociado con calcinosis en ganado en Papua, Nueva Guinea. Cestrum diurnum ha sido asociado como el causante de la enfermedad en Hawaii y Jamaica. Y Solanum sodomaeum ha sido mencionado en procesos de la enfermedad en Hawaii. En Brasil ha sido reconocida desde 1968 y descrita en granjas de la municipalidad de Julio de Castihos, Río Grande do Sul; y la enfermedad ha sido reproducida experimentalmente en conejos y ovinos mediante la administración de Nierembergia veitchii (Zanuzzi et al., 2008; Zanuzzi et al., 2012; García et al., 2012; Rissi et al., 2009).

Trisetum flavescens es un vegetal que puede ser ingerido en cualquier estado, incluso estando seco, por lo cual puede ser causa de intoxicación para los animales al momento de salir a pastorear. El cuadro se caracteriza por calcinosis, con deposición de calcio en los tejidos blandos, como es el caso de músculos y tendones, corazón y grandes arterias, incluída la aorta (Jennings, 1969; Cuesta, 2003). 
En el presente estudio se describe el caso clínico de un ovino que de acuerdo a antecedentes y datos emitidos por el propietario, el animal había estado ingiriendo Trisetum flavescens, y mostró signos clínicos de decaimiento, entre otros; las lesiones más evidentes al estudio macro y microscópicas se asociaron a metaplasia cartilaginosa de aurícula derecha condición que pone de manifiesto el riesgo por el consumo de plantas, las cuales pueden contener una variedad de sustancias que pueden afectar y deteriorar la salud de diferentes especies domésticas herbívoras.

\section{Historia clínica}

De un total de 25 ovinos en la unidad de producción, se remitió un ovino macho de 2.5 años de edad; con un cuadro clínico de disminución del apetito, pérdida de peso, distención abdominal y postración; la única información proporcionada por el Médico Veterinario Zootecnista sobre el animal, es que fue desparasitado con albendazol 5 días antes de mostrar la signología referida; su dieta era a base de rastrojo de maíz, soya, canola, sorgo y pre-mezcla, no presentaba fiebre y a la auscultación cardíaca se escuchaba ruido de galope tenue, fue medicado por el MVZ sin mejoría con Estreptomicina y Meglumina de Flunixin.

Se solicitó más información al propietario sobre el manejo del rebaño y en particular sobre el consumo de otro tipo de alimento para los ovinos, refiriendo que los animales regularmente pastoreaban alrededor de 3-4 horas al día, señalando que en el área de pastoreo crecía una hierba de 60 a 90 centímetros de altura, con inflorescencia estrecha color amarillo verdoso a púrpura, que al paso del tiempo mostraba una tonalidad amarillo dorado brillante; que era ingerida por el ovino examinado y otros animales del rebaño en forma común y en buena cantidad (también notificó que el animal del caso fue el único que mostró signos clínicos), por lo cual se le solicitó una muestra de la planta, para su identificación taxonómica.

\section{MÉTODO}

Se colectó sangre completa del ovino en tubos sin anticoagulante y se sacrificó de acuerdo con lo establecido por la SAGARPA (1995), Se realizó el estudio anatomopatológico, para valorar los cambios macroscópicos; se colectaron muestras de aurículas y corazón entre otros tejidos, que fueron fijados en formol bufferado al 10\% (10:1); para la valoración histológica se usó la tinción de hematoxilina-eosina.

Las muestras de la planta se enviaron al herbario de la Universidad Nacional Autónoma de México para su identificación taxonómica.

\section{RESULTADOS}

\section{Estudio anatomopatológico}

Hallazgos macroscópicos relevantes: en corazón se observó degeneración mucoide de la grasa coronaria y la aurícula derecha presentó nodulaciones duras al tacto de 
color blanquecino (Figura 1 y 2). Se presentaron adherencias pleurales, congestión hipostática, hemoperitoneo, distención de retículo, rumen, omaso y abomaso. El diagnóstico presuntivo fue el de metaplasia cartilaginosa de aurícula derecha.

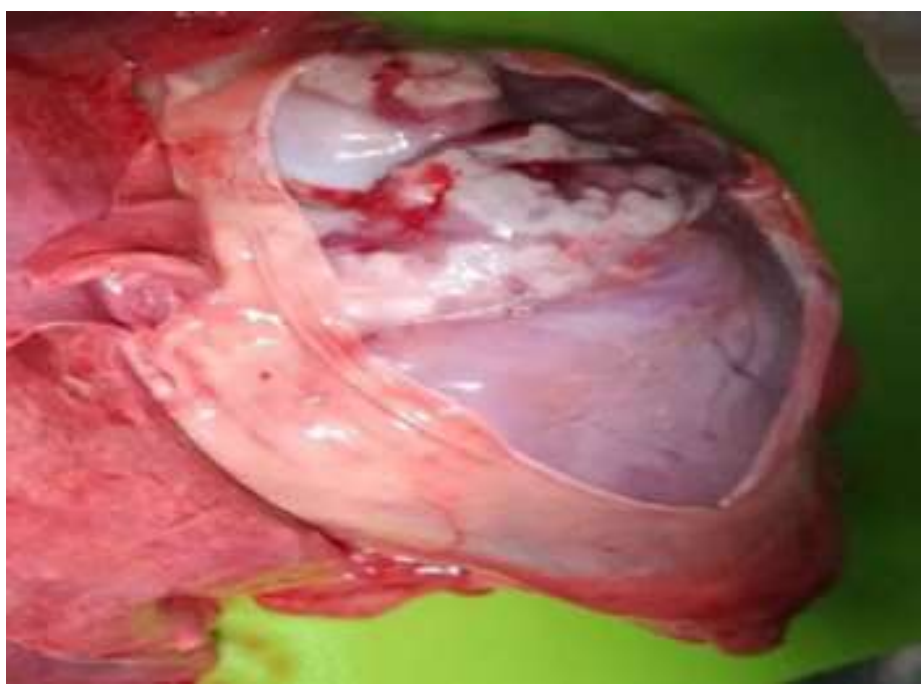

Figura 1. Corte de pericardio. Hidropericardio ligero, pericardio engrosado y de apariencia opaca.

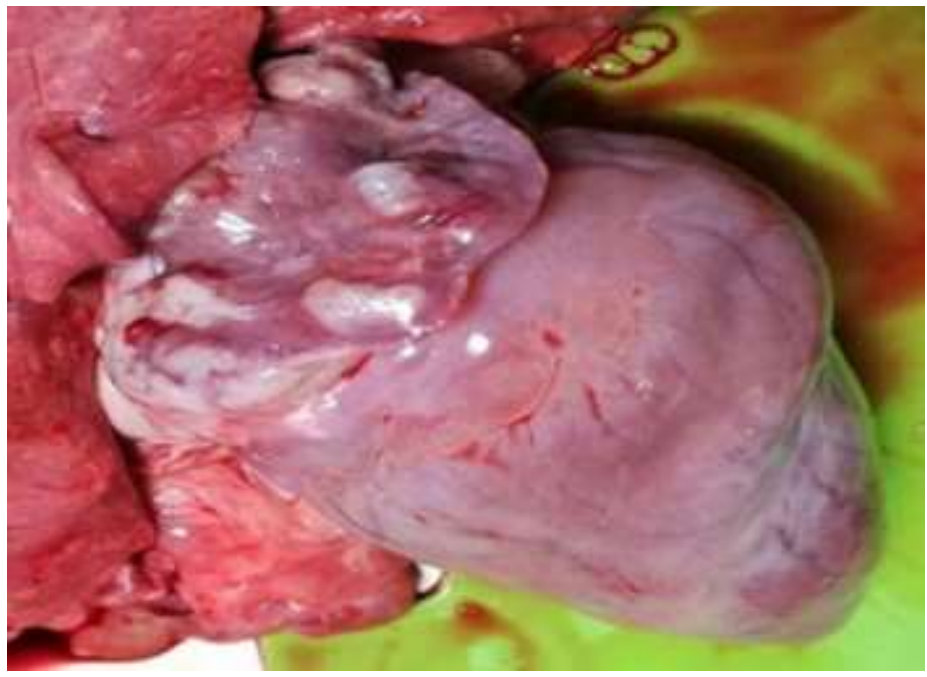

Figura 2. Corazón. Contorno redondeado, con degeneración mucoide de grasa, áreas blanquecinas en aurícula derecha (duras tanto al tacto como al corte).

\section{Estudio histopatológico}

El hallazgo más significativo al estudio microscópico de la aurícula derecha fue la metaplasia cartilaginosa en donde se observa cartílago hialino y en los bordes de la aurícula áreas de osificación del miocardio (Figuras 3 y 4). 


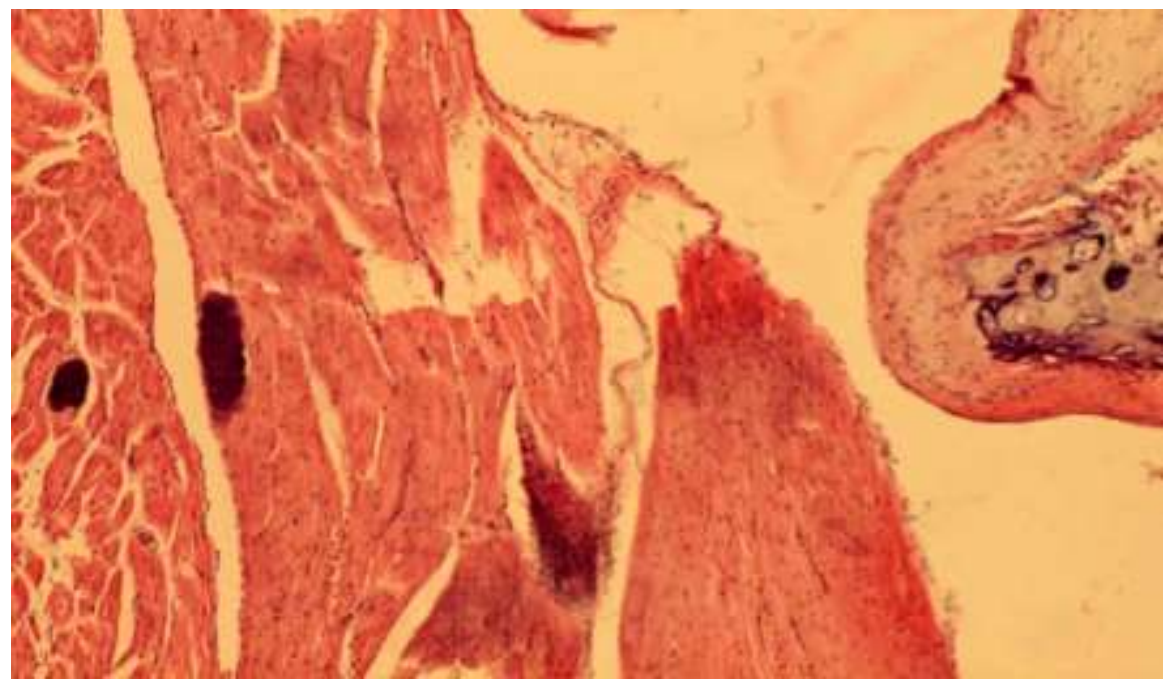

Figura 3. Corte histológico de pared de corazón. Se observa tumefacción y ondulación de fibras musculares, presencia de Sarcocystis; sustitución de miocitos por condrocitos en músculo cardiaco. Tinción H\&E. 40X.

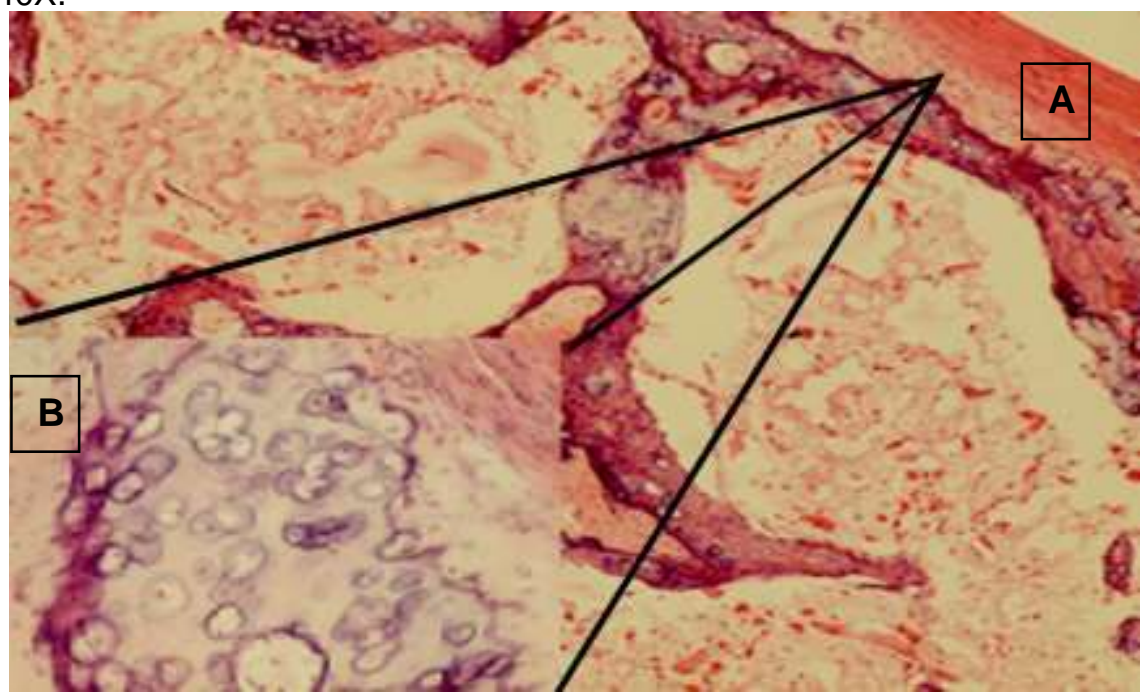

Figura 4. Corte histológico de aurícula derecha. A. Condrocitos que han sustituido al músculo cardiaco. 4X. B. Lagunas ocupadas por condrocitos hipertrofiados (imagen en relieve). 100X.Tinción H\&E.

Se envió muestra de sangre completa sin anticoagulante, para prueba complementaria de bioquímica clínica, la cual indicó una hiperfosfatemia e hipermagnesemia (Tabla 1).

Tabla 1. Resultado de bioquímica clínica del ovino

\begin{tabular}{ccc}
\hline Analito & Valor obtenido & Valor de referencia \\
\hline Calcio & $2.2 \mathrm{mmol} / \mathrm{l}$ & $2.25-2.6 \mathrm{mmol} / \mathrm{l}$ \\
Fósforo & $7.51 \mathrm{mmol} / \mathrm{l}$ & $1.40-2.40 \mathrm{mmol} / \mathrm{l}$ \\
Magnesio & $1.29 \mathrm{mmol} / \mathrm{l}$ & $0.9-1.14 \mathrm{mmol} / \mathrm{l}$ \\
\hline
\end{tabular}




\section{Identificación taxonómica de la planta}

El herbario de la Universidad Nacional Autónoma de México, identificó a la planta como Trisetum flavescens (L.) P. Beauv. (IBUNAM:MEXU:1470131).

\section{DISCUSIÓN}

El hallazgo más relevante del estudio clínico patológico en el ovino, fue lo observado en el corazón, por lo que se llegó al diagnóstico definitivo de metaplasia cartilaginosa auricular que se asoció a la ingestión de Trisetum flavescens, mejor conocida como "avena dorada" vegetal que resulta ser tóxico para el ganado; lo cual fue reafirmado y considerado en el historial clínico detectado a la auscultación, forma de presentación del proceso y por los datos del consumo de la planta por el animal. Dirksen et al. (2003), al valorar a diferentes ovinos de un rebaño que permanecían en una pradera consumiendo Trisetum flavenscens observaron en varios ovinos la cojera típica de la calcinosis (carpo ligeramente doblado y posición relativamente estirada de las articulaciones tarsal y del espolón al estar de pie, "arrodillado" en el carpo), con un deterioro progresivo de los sistemas circulatorio y respiratorio (soplo endocárdico holosistólico, congestión y disnea espiratoria). En contraste, cabe mencionar, que como único caso patológico que se presentó en la unidad de producción ovina, el MVZ que atiende a estos animales, reportó que a la auscultación cardiaca escuchó ruido de galope tenue.

La metaplasia cartilaginosa inicial de miocardio asociada al consumo de Trisetum flavenscens puede tener y mostrar signos y lesiones variables, como lo refieren Dirksen et al. (2003), Reilly et al. (2012) y Scott (2007), que consideran que la metaplasia cartilaginosa de las válvulas cardíacas, el endocardio y los vasos arteriales en la mayoría de los casos son graves; y que a la evaluación patomorfológica son consistentes con la insuficiencia cardiovascular (Cebra y Cebra, 2012; Brounts et al., 2005); otros hallazgos relevantes son la calcificación de varios tendones y ligamentos, riñones y en casos crónicos del parénquima pulmonar (Ribeiro et al., 2017; Estepa et al., 2006).

Al considerar el dato proporcionado por el propietario de que el animal consumía Trisetum flavescens, se relacionó a un proceso de intoxicación, aunque no se pudo determinar la cantidad de ingestión por día, al menos llevaba alrededor de un mes y medio a dos meses consumiendo la planta. En la valoración a la necropsia del ovino de este estudio, no se observaron otras lesiones significativas en otros tejidos, solo lo hallado en el corazón; lo cual a la vez es relevante por el poco reporte de casos similares. La concentración de vitamina D en Trisetum flavescens es de 600 a $800 \mathrm{UI}$ $\mathrm{kg} / \mathrm{MS}$. Mello (2003) y Jennings (1969), refieren que, con las alteraciones bioquímicas, consecuentemente se presentan cambios morfológicos, principalmente calcificaciones 
en fibras elásticas y membranas basales de aquellos tejidos con tendencia a calcificarse (corazón, arterias y riñones entre otros tejidos).

A través de la bioquímica realizada, la hiperfosfatemia e hipermagnesemia son datos de relevancia para considerar en la orientación diagnóstica del caso; el desbalance de este tipo de analitos puede ocasionar trastornos específicos en el metabolismo de otros compuestos de importancia fisiológica, como el calcio, la periodicidad del consumo del vegetal identificado determinaría la signología y patología; además, se debe considerar la variación individual de cada organismo en respuesta al desequilibrio de fósforo- calcio - magnesio ( y posiblemente vitamina $D$ y $K$ ), ya que de acuerdo a su movilidad o deposición inferirán en el desequilibrio u alteración funcional como en el caso analizado. En la información referida por Scott (2007), menciona que las concentraciones séricas de calcio y fósforo aumentan entre un $20 \%$ y un $25 \%$, con aumentos de hasta $3.4 \mathrm{mmol} / \mathrm{l}$ de calcio y $4 \mathrm{mmol} / \mathrm{l}$ de fósforo; $y$ ante la frecuencia de este tipo de problemas sugiere la evaluación radiológica, en donde puede detectarse calcificación tisular.

Los animales en pastoreo en áreas problema presentan hipercalcemia e hiperfosfatemia, la expresión "in situ" de osteopontina parecería ser de particular importancia en la génesis de las calcificaciones ya que esa proteína se une muy fuertemente a hidroxiapatita y por lo tanto, presenta un rol importante en el proceso de calcificación, la enfermedad hace su aparición en verano y otoño, especialmente en épocas de sequía (Mello, 2003; Reilly et al., 2012).

Los animales afectados se mueven a desgano, con pasos cortos y rígidos, los animales enfermos al moverlos presentan disnea y taquicardia, y pueden caer al suelo con signos de insuficiencia cardiaca y pulmonar. Al levantarse, lo hacen con dificultad en casos subclínicos; para su correcto diagnóstico diferencial, podría resultar de utilidad la determinación de los valores séricos de calcio y fósforo para la determinación de osteocalcina, osteopontina y de otras proteínas inducidas por el efecto de la vitamina $D$ en la diferenciación celular. Tan solo 12 gramos semanales de hojas de Trisetum flavescens alcanzan para reproducir la enfermedad en una vaca de $300 \mathrm{~kg}$. En 4 meses; un bovino que ingirió 50 hojas por día, se presentó clínicamente enfermo en 8 a 10 semanas (Cuesta, 2003; Machado et al., 2020).

Este problema se ha presentado desde hace unos cuantos años a nivel mundial, afectando hasta la actualidad en primera instancia al continente americano. La calcinosis enzoótica de Europa Central es originada por la intoxicación crónica por la gramínea conocida como avena dorada (Trisetum flavescens), considerada hasta hace unos años como una forrajera de gran valor. Los otros vegetales de reconocida capacidad calcinogénica, Cestrum diurnum, Solanum torvum, Nierembergia veitchii y 
Stenotaphrum secundatum ocasionan la enfermedad en áreas limitadas (Garcia et al., 2012; Gupta, 2012).

En Alemania y Austria la calcinosis enzoótica bovina es provocada por la avena dorada (Trisetum flavescens) que crece en la zona alpina y es mucho menos tóxica que el duraznillo blanco (S. malacoxylon), ya que tiene que integrar las pasturas en un 2025\% para provocar toxicidad (Waser et al., 1983; Dallorso et al., 2001; Zanuzzi et al., 2012; Braun et al., 2000; Gupta, 2012).

De acuerdo con la valoración de Rissi et al. (2009), refieren que la calcinosis enzoótica de los animales domésticos ha sido descrita en varias partes del mundo. Diversas plantas poseen dichas propiedades calcinogénicas. En bovinos se ha demostrado que la enfermedad es producida por la ingestión de Solanum malacoxylon en Argentina, Brasil y Uruguay; mientras que en Cuba el Cestrum diurnum está diseminado en toda la isla, que ocasiona la enfermedad en temporada de sequía.

Mientras que la presentación de la calcinosis enzoótica en Sudamérica, coincide con el área de distribución de Trisetum flavescens resulta un problema muy serio en la Cuenca del Río Salado en Buenos Aires, pero está presente en toda la Cuenca de la Plata. La incidencia de esta enfermedad es muy difícil de estimar debido a la existencia de numerosos casos subclínicos, se ha estimado en un $10 \%$ en Buenos Aires y en un 8.2\% en Santa Fe (Zanuzzi et al., 2012; Rissi et al., 2009).

Las amplias regiones de producción animal que tiene México hacen propicio el desarrollo de una diversidad de vegetales que crecen naturalmente, los cuales pueden contener a la vez una variada cantidad de sustancias que deben determinarse y evaluarse, esto con la finalidad de minimizar la presentación de casos de intoxicación en las diferentes especies domésticas herbívoras, con una consecuente alteración morfológica y funcional como la referida en este estudio.

\section{CONCLUSIÓN}

La metaplasia cartilaginosa de aurícula derecha observada en el ovino se asoció al elevado consumo de la planta tóxica "avena dorada" (Trisetum flavescens), la cual puede crecer en diferentes terrenos; y ser un riesgo de toxicosis para diferentes especies animales y ocasionar alteraciones significativas e incluso la muerte.

\section{LITERATURA CITADA}

BRAUN U, Diener M, Camenzind D, Flückiger M, Thoma R. 2000. Enzootic calcinosis in goats caused by golden oat grass (Trisetum flavescens). Vet Rec. 146(6):161-162. http://dx.doi.org/10.1136/vr.146.6.161 
BROUNTS SH, Baird AN, Baird DK. 2005. What is your diagnosis? Dystrophic mineralization. $J \quad A m$ Vet Med Assoc. 226(3):349-50. https://doi.org/10.2460/javma.2005.226.349

CEBRA Ch, Cebra M. 2012. Diseases of the Cardiovascular System. Sheep and Goat Medicine. $2^{\underline{a}}$ ed. Elsevier Saunders. Pp. 503-506.ISBN: 9781455754854

CUESTA GDIF. 2003. Patología veterinaria. Colombia. Universidad de Antioquia. http://www.worldcat.org/title/patologia-veterinaria/oclc/63761873

DALLORSO ME, Gil S, Pawlak E, Lema F, Márquez A. 2001. 1,25(OH)2 vitamin D concentration in the plasma of Solanum glaucophyllum intoxicated rabbits. Aust Vet $\mathrm{J}$. 79(6):419-23. https://doi.org/10.1111/j.1751-0813.2001.tb12987.x

DIRKSEN G, Sterr K, Hermanns W. 2003. Enzootic calcinosis in sheep after consumption of golden oat grass (Trisetum flavescens L., P. B.). Dtsch Tierarztl Wochenschr. 110(12):475-483. https://pubmed.ncbi.nlm.nih.gov/14746053/

ESTEPA JC, Aguilera-Tejero E, Zafra R, Mayer-Valor R, Rodríguez M, Pérez J. 2006. An unusual case of generalized soft-tissue mineralization in a suckling foal. Vet Pathol. 43(1):64-7. https://doi.org/10.1354/vp.43-1-64

GARCÍA y SANTOS C, Pereira R, Etcheberry G, Goyen JM, Pérez W, Capelli A, Alonso E, Ruiz-Díaz A, Riet-Correa F. 2012. Enzootic calcinosis caused by Nierembergia rivularis in sheep. $J$ Vet Diagn Invest. 24(2):423-6. https://doi.org/10.1177/1040638711435143

GRABNER A, Kraft W, Essich G, Hänichen T. 1985. Enzootic calcinosis in the horse. Tierarztl Prax. 1:84-93. https://pubmed.ncbi.nlm.nih.gov/4012785/

GUPTA RC. 2012. Veterinary Toxicology: Basic and Clinical Principles. Academic Pres. San Diego, California. U.S.A. Pp. 172. https://doi.org/10.1016/C2010-0-67763-7

JENNINGS IW. 1969. Algunas cardiomiopatías de los animales un análisis indicador de posibles analogías con enfermedades del hombre. Boletín de la Oficina Sanitaria Panamericana. Pp 108-119.

https://iris. paho.org/bitstream/handle/10665.2/12700/v67n2p108.pdf?sequence=1\&is Allowed $=\mathrm{y}$ 
MACHADO M, Castro MB, Gimeno EJ, Barros SS, Riet-Correa F. 2020. Enzootic calcinosis in ruminants: A review. Toxicon. 187:1-9. https://doi.org/10.1016/j.toxicon.2020.08.009

MELLO JR. 2003. Calcinosis-calcinogenic plants. Toxicon. 41(1):1-12. https://doi.org/10.1016/S0041-0101(02)00241-6

ODRIOZOLA ER, Rodríguez AM, Micheloud JF, Cantón GJ, Caffarena RD, Gimeno EJ, Bodega JJ, Gardey P, Iseas FB, Giannitti F. 2018. Enzootic calcinosis in horses grazing Solanum glaucophyllum in Argentina. J Vet Diagn Invest. 30(2):286-289. https://doi.org/10.1177/1040638717746447

REILLY KL, Baird AN, Pugh DG. 2012. Diseases of the Musculoskeletal System. Sheep and Goat Medicine. 2 $2^{\mathrm{a}}$ ed. Elsevier Saunders. Pp. 306-307. ISBN: 9781455754854

RIBEIRO M, Borges AP, Curtio LM, Bianchi IN, Magalhães AO, Pereira AHB, Colodel EM, Furlan FH. 2017. Calcinose Enzoótica em Ovinos no Pantanal Matogrossense. Pesquisa Veterinária Brasileira. 37:13-15. http://www.pvb.com.br/portal/download_artigo/MjEOMnwyMDIwMTExOTIzNTU0Mg==

RISSI RD, Brown CC, Barros LC. 2009. Chronic and acute clinical manifestations associated with systemic mineralization caused by ingestion of Nierembergia veitchii in sheep in southern Brazil. Small Ruminant Res. 87: 102-104. https://doi.org/10.1016/j.smallrumres.2009.09.035

SAGARPA (Secretaría de Agricultura, Ganadería, Desarrollo Rural, Pesca y Alimentación). 1995. NOM-033-ZOO-1995. Sacrificio humanitario de los animales domésticos y silvestres. México: Diario Oficial de la Federación; 1997. http://dof.gob.mx/nota_detalle.php?codigo=4864926\&fecha=22/01/1997

SCOTT RP. 2007. Cardiovascular System. Sheep Medicine. Manson Publishing - The Veterinary Press. Reino Unido. 161-164. https://books.google.com.mx/books?id=whhp7rlQru0C\&printsec=frontcover\&hl=es\&s ource=gbs_ge_summary_r\&cad=0\#v=onepage \&q\&f=false

SUN J. 2010. Vitamin D and mucosal immune function. Curr Opin Gastroenterol. 26(6):591-595. https://doi.org/10.1097/MOG.0b013e32833d4b9f 
WASER J, Meyer J, Hänichen T, Dirksen G. 1983. Trisetum flavescens and vitamin D3: comparison of the calcinogenic effect in sheep. Berl Munch Tierarztl Wochenschr. 96(5):163-166. https://pubmed.ncbi.nlm.nih.gov/6307254/

WU S, Sun J. 2011. Vitamin D, vitamin D receptor, and macroautophagy in inflammation and infection. Discov Med. 11(59):325335. https://www.ncbi.nlm.nih.gov/pmc/articles/PMC3285235/

ZANUZZI CN, Nishida F, Portiansky EL, Fontana PA, Gimeno EJ, Barbeito CG. 2012. Effects of Solanum glaucophyllum toxicity on cell proliferation and apoptosis in the small and large intestine of rabbits. Res. In Vet. Sci. 93:336-342. https://doi.org/10.1016/j.rvsc.2011.07.018

ZANUZZI CN, Fontana PA, Barbeito CG, Portiansky EL, Gimeno EJ. 2008. Paneth cells: histochemical and morphometric study in control and Solanum glaucophyllum intoxicated rabbits. Eur J Histochem. 52(2):93-100. https://doi.org/10.4081/1193 